ThE AstrophysiCAL JouRNAL, 465:965-971, 1996 July 10

(C) 1996. The American Astronomical Society. All rights reserved. Printed in U.S.A.

\title{
Ne v AND Ne VI LINES IN THE ULTRAVIOLET SPECTRUM OF THE SYMBIOTIC STAR RR TELESCOPII
}

\author{
B. ESPEY ${ }^{1,2}$ \\ Center for Astrophysical Sciences, Department of Physics and Astronomy, Johns Hopkins University, Baltimore, MD 21218; \\ espey@pha.jhu.edu \\ F. P. KeEnan AND F. C. McKenna \\ Department of Pure and Applied Physics, The Queen's University of Belfast, Belfast BT7 1NN, Northern Ireland, UK; \\ f.keenan@qub.ac.uk; f.mckenna@qub.ac.uk \\ W. A. Feibelman ${ }^{3}$ \\ Laboratory for Astronomy and Solar Physics, Code 684.1, NASA/Goddard Space Flight Center, Greenbelt, MD 20771; \\ feibelman@iue.gsfc.nasa.gov \\ AND
}

K. M. Aggarwal

Department of Physics and Astrophysics, University of Delhi, Delhi 110007 , India

Received 1995 October 4; accepted 1996 January 25

\begin{abstract}
New theoretical $\mathrm{Ne}$ VI] electron density-sensitive ratios are presented for the intercombination transitions $R_{1}=I(1006.1 \AA) / I(999.6 \AA)$ and $R_{2}=I(1010.6 \AA) / I(999.6 \AA)$. Temperature-sensitive ratios are also given for the Ne v] ratio $R=I(1137.0 \AA) / I(1574.8 \AA)$. We discuss the potential usefulness of these line ratios for studying hot gas and apply them to the case of the symbiotic system RR Telescopii. Using far-UV data that has recently been acquired with the Hopkins Ultraviolet Telescope, we conclude that the $\mathrm{Ne} \mathrm{V}$ and $\mathrm{Ne}$ VI emission in RR Tel is due to photoionization, with the temperature and density of the emitting region being approximately $T_{e} \simeq 18,000 \mathrm{~K}$ and $N_{e} \simeq 10^{6} \mathrm{~cm}^{-3}$, respectively. These results are similar to those estimated using other high ionization line diagnostics of the nebular material, such as O v $I(1371 \AA) / I(1644 \AA)$ and Ne IV] $I(1602 \AA) / I(2423 \AA)$. Although these Ne v] and Ne vI] lines have been used as diagnostics for solar physics, we believe that this is the first instance in which they have been applied to another astrophysical object.
\end{abstract}

Subject headings: atomic processes - binaries: symbiotic - stars: individual (RR Telescopii) ultraviolet: stars

\section{INTRODUCTION}

Emission lines arising from the $2 s^{2} 2 p^{2} P-2 s 2 p^{2}{ }^{4} P$ intercombination transitions in the B-like ions $\mathrm{C}$ II, $\mathrm{N}$ III, and $\mathrm{O}$ IV have been frequently detected in the spectra of gaseous nebulae, including symbiotic stars and planetary nebulae (see, for example, Keenan et al. 1993, 1994). The potential usefulness of these lines as electron density diagnostics for the emitting plasma was first noted by Flower \& Nussbaumer (1975a), who presented theoretical line ratios for O IV applicable to solar observations. Subsequently, Stencel et al. (1981), Czyzak, Keyes, \& Aller (1986), and Nussbaumer \& Storey (1982) derived results for C II, N III, and O IV, respectively, which were applicable to the lower density plasmas found in nebulae.

Although intercombination lines from higher atomic number B-like ions, such as Ne VI, have been extensively detected in solar spectra (see, for example, Keenan et al. 1995), to the best of our knowledge they have never been observed in nonsolar astronomical sources. However, recent observations of the symbiotic star RR Telescopii with the Hopkins Ultraviolet Telescope (HUT) have revealed numerous emission lines in the spectrum between

\footnotetext{
${ }^{1}$ Also Department of Physics and Astronomy, University of Pittsburgh, Pittsburgh, PA 15260.

${ }^{2}$ Guest investigator, Astro-2 mission.

${ }^{3}$ Guest observer with the International Ultraviolet Explorer (IUE) satellite, which is operated and sponsored by NASA, by the European Space Agency, and by the Science and Engineering Research Council of the United Kingdom.
}

820 and $1840 \AA$, including the Ne VI intercombination transitions near $\sim 1000 \AA$ (Espey et al. 1995). In this paper, we present theoretical ratios involving these lines and use the HUT data to investigate their usefulness as plasma diagnostics. In addition, we present HUT measurements of $\mathrm{Ne} \mathrm{v}$ lines in the RR Tel spectrum, which should also be useful as temperature diagnostics, plus IUE data for $\mathrm{Ne} \mathrm{v}$ transitions, which allow the extinction toward RR Tel to be accurately derived. The latter is vital for determining dereddened line fluxes, necessary for the evaluation of ratios.

\section{ATOMIC DATA AND THEORETICAL RATIOS}

The model ion for $\mathrm{Ne} v$ consisted of the nine energetically lowest $L S$ states, namely, $2 s^{2} 2 p^{2}{ }^{3} P,{ }^{1} D,{ }^{1} S$ and $2 s 2 p^{3}{ }^{5} S,{ }^{3} D$, ${ }^{3} \mathrm{P},{ }^{1} \mathrm{D},{ }^{3} \mathrm{~S},{ }^{1} \mathrm{P}$, making a total of 15 fine-structure levels. Energies for these levels were taken from Moore (1971).

Electron impact excitation rates among the $2 s^{2} 2 p^{2}$ levels in Ne v were obtained from Lennon \& Burke (1994), data for all other transitions being taken from Aggarwal (1986). For Einstein $A$-coefficients, the calculations of Nussbaumer $\&$ Rusca (1979) and Aggarwal (1986) for $2 s^{2} 2 p^{2}-2 s^{2} 2 p^{2}$ and $2 s^{2} 2 p^{2}-2 s 2 p^{3}$ transitions, respectively, were adopted, apart from the value for $2 s^{2} 2 p^{2}{ }^{3} P_{0}-2 s^{2} 2 p^{2}{ }^{3} P_{1}$, where the result of Johnson, Kingston, \& Dufton (1984) was preferred. Proton collisional excitation rates, which are only important within the $2 s^{2} 2 p^{2}{ }^{3} P$ ground term, were obtained from Faucher, Masnou-Seeuws, \& Prudhomme (1980).

The Ne vi model ion consisted of the eight energetically lowest $L S$ states, namely, $2 s^{2} 2 p^{2} P ; 2 s 2 p^{2}{ }^{4} P,{ }^{2} D,{ }^{2} S,{ }^{2} P$; and 
$2 p^{3}{ }^{4} S,{ }^{2} \mathrm{D},{ }^{2} \mathrm{P}$, making a total of 15 fine-structure levels. Energies for these were obtained from Moore (1971).

Electron impact excitation rates for transitions in Ne VI were taken from Zhang, Graziani, \& Pradhan (1994), while for Einstein $A$-coefficients the calculations of Dankworth \& Trefftz (1978) were adopted, apart from the value for $2 s^{2} 2 p^{2} P_{1 / 2}-2 s^{2} 2 p^{2} P_{3 / 2}$, where we interpolated the calculations of Lennon et al. (1985), Nussbaumer \& Storey (1982), and Flower \& Nussbaumer (1975b) for C II, O IV, and Na VII, respectively. Excitation by proton impact will be important for this transition (Seaton 1964), and in the present analysis we have used the rates of Foster, Reid, \& Keenan (1996a). However, Flower \& Nussbaumer (1975a) have pointed out that proton excitation should also be included for the $2 s 2 p^{2}{ }^{4} P_{J}-2 s 2 p^{2}{ }^{4} P_{J^{\prime}}$ transitions, and for these rates we have adopted the recent calculations of Foster, Reid, \& Keenan (1996b).
Using the atomic data discussed above in conjunction with the statistical equilibrium code of Dufton (1977), relative $\mathrm{Ne} \mathrm{V}$ and $\mathrm{Ne}$ vi level populations, and hence emissionline strengths, were generated for a range of electron temperatures and densities. Further details of the procedures involved and approximations made may be found in Dufton (1977) and Dufton et al. (1978). Given that all of the adopted atomic data have estimated uncertainties of typically $\pm 10 \%$ (see individual references), we believe that our derived theoretical line ratios should be accurate to better than $\pm 20 \%$.

Tables 1-3 contain the values of the diagnostic ratios $R$, $R_{1}$, and $R_{2}$ as a function of electron density for several temperatures in the range $T_{e}=10,000-250,000 \mathrm{~K}$, appropriate to both nebular- and coronal-type plasmas. A more immediate appreciation of the diagnostic power of these ratios may be achieved by examining Figures $1-3$, which

TABLE 1

Ne v] $R$ Ratios

\begin{tabular}{lccccccr}
\hline \hline & \multicolumn{7}{c}{$T_{e}(\mathrm{~K})$} \\
\cline { 2 - 8 } $\log N_{e}$ & 10,000 & 15,000 & 10,000 & 30,000 & 40,000 & 100,000 & 250,000 \\
\hline $3.0 \ldots \ldots$ & 0.116 & 0.380 & 0.682 & 0.996 & 1.35 & 2.18 & 2.21 \\
$3.5 \ldots \ldots$ & 0.116 & 0.380 & 0.682 & 0.996 & 1.34 & 2.18 & 2.21 \\
$4.0 \ldots \ldots$ & 0.116 & 0.380 & 0.682 & 0.995 & 1.34 & 2.18 & 2.21 \\
$4.5 \ldots \ldots$ & 0.116 & 0.380 & 0.682 & 0.992 & 1.34 & 2.18 & 2.21 \\
$5.0 \ldots \ldots$ & 0.115 & 0.379 & 0.681 & 0.985 & 1.33 & 2.17 & 2.20 \\
$5.5 \ldots \ldots$ & 0.110 & 0.367 & 0.663 & 0.962 & 1.30 & 2.14 & 2.18 \\
$6.0 \ldots \ldots$ & 0.0994 & 0.337 & 0.612 & 0.899 & 1.23 & 2.06 & 2.12 \\
$6.5 \ldots \ldots$ & 0.0807 & 0.278 & 0.508 & 0.765 & 1.06 & 1.85 & 1.97 \\
$7.0 \ldots \ldots$ & 0.0615 & 0.210 & 0.383 & 0.583 & 0.816 & 1.48 & 1.65 \\
$7.5 \ldots \ldots$. & 0.0558 & 0.181 & 0.318 & 0.471 & 0.647 & 1.13 & 1.26 \\
$8.0 \ldots \ldots$. & 0.0733 & 0.222 & 0.373 & 0.519 & 0.680 & 1.05 & 1.08 \\
$8.5 \ldots \ldots$ & 0.144 & 0.414 & 0.668 & 0.869 & 1.08 & 1.43 & 1.29 \\
$9.0 \ldots \ldots$ & 0.375 & 1.04 & 1.64 & 2.05 & 2.47 & 2.89 & 2.33 \\
$9.5 \ldots \ldots$. & 1.10 & 3.02 & 4.72 & 5.79 & 6.86 & 7.61 & 5.77 \\
$10.0 \ldots \ldots$. & 3.30 & 9.09 & 14.2 & 17.4 & 20.6 & 22.4 & 16.6 \\
$10.5 \ldots \ldots$. & 9.62 & 26.7 & 42.1 & 52.5 & 62.0 & 67.5 & 49.9 \\
$11.0 \ldots \ldots$ & 24.6 & 70.5 & 113 & 148 & 176 & 196 & 147 \\
$11.5 \ldots \ldots$ & 48.6 & 147 & 246 & 350 & 427 & 498 & 393 \\
$12.0 \ldots \ldots$ & 70.4 & 223 & 389 & 617 & 776 & 974 & 836 \\
\hline
\end{tabular}

TABLE 2

$\mathrm{Ne}$ VI] $R_{1}$ RATIOS

\begin{tabular}{cccccccc}
\hline \hline & \multicolumn{7}{c}{$T_{\boldsymbol{e}}(\mathrm{K})$} \\
\cline { 2 - 8 } $\log N_{e}$ & 10,000 & 15,000 & 10,000 & 30,000 & 40,000 & 100,000 & 250,000 \\
\hline $3.0 \ldots \ldots$ & 1.09 & 1.06 & 1.04 & 1.03 & 1.02 & 1.05 & 1.08 \\
$3.5 \ldots \ldots$ & 1.08 & 1.05 & 1.04 & 1.02 & 1.01 & 1.05 & 1.08 \\
$4.0 \ldots \ldots$ & 1.06 & 1.03 & 1.02 & 1.01 & 1.00 & 1.04 & 1.07 \\
$4.5 \ldots \ldots$ & 0.995 & 0.979 & 0.973 & 0.969 & 0.972 & 1.02 & 1.05 \\
$5.0 \ldots \ldots$ & 0.885 & 0.879 & 0.880 & 0.885 & 0.899 & 0.960 & 1.00 \\
$5.5 \ldots \ldots$ & 0.772 & 0.765 & 0.766 & 0.772 & 0.788 & 0.847 & 0.894 \\
$6.0 \ldots \ldots$ & 0.707 & 0.693 & 0.689 & 0.688 & 0.693 & 0.725 & 0.758 \\
$6.5 \ldots \ldots$ & 0.680 & 0.663 & 0.655 & 0.648 & 0.644 & 0.650 & 0.662 \\
$7.0 \ldots \ldots$ & 0.671 & 0.652 & 0.642 & 0.634 & 0.625 & 0.618 & 0.619 \\
$7.5 \ldots \ldots$ & 0.668 & 0.648 & 0.638 & 0.629 & 0.619 & 0.608 & 0.603 \\
$8.0 \ldots \ldots$ & 0.666 & 0.646 & 0.637 & 0.627 & 0.616 & 0.604 & 0.598 \\
$8.5 \ldots \ldots$ & 0.664 & 0.644 & 0.635 & 0.625 & 0.615 & 0.602 & 0.596 \\
$9.0 \ldots \ldots$ & 0.656 & 0.639 & 0.630 & 0.621 & 0.611 & 0.599 & 0.593 \\
$9.5 \ldots \ldots$ & 0.636 & 0.622 & 0.615 & 0.609 & 0.600 & 0.592 & 0.588 \\
$10.0 \ldots \ldots$ & 0.582 & 0.577 & 0.575 & 0.576 & 0.569 & 0.571 & 0.572 \\
$10.5 \ldots \ldots$ & 0.475 & 0.481 & 0.487 & 0.498 & 0.496 & 0.517 & 0.531 \\
$11.0 \ldots \ldots$ & 0.345 & 0.353 & 0.362 & 0.378 & 0.379 & 0.415 & 0.444 \\
$11.5 \ldots \ldots$ & 0.259 & 0.261 & 0.265 & 0.273 & 0.274 & 0.301 & 0.328 \\
$12.0 \ldots \ldots$ & 0.223 & 0.220 & 0.220 & 0.221 & 0.220 & 0.231 & 0.245 \\
\hline
\end{tabular}


TABLE 3

$\mathrm{Ne}$ vI] $R_{\mathbf{2}}$ RATIOS

\begin{tabular}{cccccccc}
\hline \hline & \multicolumn{7}{c}{$T_{e}(\mathrm{~K})$} \\
\cline { 2 - 7 } $\log N_{e}$ & 10,000 & 15,000 & 10,000 & 30,000 & 40,000 & 100,000 & 250,000 \\
\hline $3.0 \ldots \ldots$ & 0.360 & 0.342 & 0.333 & 0.325 & 0.324 & 0.340 & 0.353 \\
$3.5 \ldots \ldots$ & 0.356 & 0.339 & 0.331 & 0.323 & 0.323 & 0.340 & 0.352 \\
$4.0 \ldots \ldots$ & 0.345 & 0.331 & 0.324 & 0.317 & 0.318 & 0.336 & 0.349 \\
$4.5 \ldots \ldots$ & 0.318 & 0.308 & 0.304 & 0.301 & 0.305 & 0.326 & 0.341 \\
$5.0 \ldots \ldots$ & 0.271 & 0.266 & 0.265 & 0.266 & 0.275 & 0.301 & 0.320 \\
$5.5 \ldots \ldots$ & 0.223 & 0.218 & 0.218 & 0.220 & 0.229 & 0.255 & 0.275 \\
$6.0 \ldots \ldots$ & 0.195 & 0.188 & 0.186 & 0.185 & 0.190 & 0.205 & 0.219 \\
$6.5 \ldots \ldots$ & 0.183 & 0.175 & 0.172 & 0.169 & 0.170 & 0.174 & 0.180 \\
$7.0 \ldots \ldots$ & 0.179 & 0.171 & 0.167 & 0.163 & 0.162 & 0.161 & 0.162 \\
$7.5 \ldots \ldots$ & 0.178 & 0.169 & 0.165 & 0.161 & 0.160 & 0.157 & 0.155 \\
$8.0 \ldots \ldots$ & 0.178 & 0.169 & 0.165 & 0.160 & 0.159 & 0.155 & 0.153 \\
$8.5 \ldots \ldots$ & 0.178 & 0.169 & 0.165 & 0.160 & 0.159 & 0.155 & 0.153 \\
$9.0 \ldots \ldots$ & 0.179 & 0.169 & 0.165 & 0.161 & 0.159 & 0.155 & 0.153 \\
$9.5 \ldots \ldots$. & 0.180 & 0.171 & 0.166 & 0.162 & 0.160 & 0.156 & 0.153 \\
$10.0 \ldots \ldots$ & 0.186 & 0.176 & 0.170 & 0.165 & 0.163 & 0.158 & 0.154 \\
$10.5 \ldots \ldots$ & 0.201 & 0.188 & 0.181 & 0.174 & 0.172 & 0.164 & 0.159 \\
$11.0 \ldots \ldots$ & 0.236 & 0.217 & 0.208 & 0.197 & 0.193 & 0.179 & 0.170 \\
$11.5 \ldots \ldots$ & 0.296 & 0.271 & 0.257 & 0.242 & 0.236 & 0.213 & 0.198 \\
$12.0 \ldots \ldots$ & 0.363 & 0.336 & 0.321 & 0.303 & 0.296 & 0.269 & 0.249 \\
\hline
\end{tabular}

present the results of Tables $1-3$, respectively. The $\mathrm{Ne} \mathrm{V}$ ratio is given by

$$
\begin{aligned}
R & =I\left(2 s^{2} 2 p^{2}{ }^{3} P_{1}-2 s 2 p^{35} S\right) / I\left(2 s^{2} 2 p^{2}{ }^{3} P_{1}-2 s^{2} 2 p^{2}{ }^{1} S\right) \\
& =I(1137.0 \AA) / I(1574.8 \AA),
\end{aligned}
$$

and the $\mathrm{Ne}$ VI line ratios

$$
\begin{aligned}
R_{1} & =I\left(2 s^{2} 2 p^{2} P_{3 / 2}-2 s 2 p^{2}{ }^{4} P_{3 / 2}\right) / I\left(2 s^{2} 2 p^{2} P_{3 / 2}-2 s 2 p^{2}{ }^{4} P_{5 / 2}\right) \\
& =I(1006.1 \AA) / I(999.6 \AA)
\end{aligned}
$$

and

$$
R_{2}=I\left(2 s^{2} 2 p^{2} P_{3 / 2}-2 s 2 p^{2}{ }^{4} P_{1 / 2}\right) / I\left(2 s^{2} 2 p^{2} P_{3 / 2}-2 s 2 p^{2}{ }^{4} P_{5 / 2}\right)
$$

$$
=I(1010.6 \AA) / I(999.6 \AA) \text {. }
$$

We note that the Ne $v$ ratio

$$
\begin{aligned}
R^{\prime} & =I\left(2 s^{2} 2 p^{2}{ }^{3} P_{2}-2 s 2 p^{35} S\right) / I\left(2 s^{2} 2 p^{2}{ }^{3} P_{1}-2 s^{2} 2 p^{2}{ }^{1} S\right) \\
& =I(1146.1 \AA) / I(1574.8 \AA)
\end{aligned}
$$

and the $\mathrm{Ne}$ VI ratio

$$
\begin{aligned}
R_{3} & =I\left(2 s^{2} 2 p^{2} P_{1 / 2}-2 s 2 p^{2}{ }^{4} P_{1 / 2}\right) / I\left(2 s^{2} 2 p^{2} P_{3 / 2}-2 s 2 p^{2}{ }^{4} P_{5 / 2}\right) \\
& =I(997.4 \AA) / I(999.6 \AA)
\end{aligned}
$$

have the same temperature and density dependence as $R$ and $R_{2}$, respectively, due to the presence of common upper

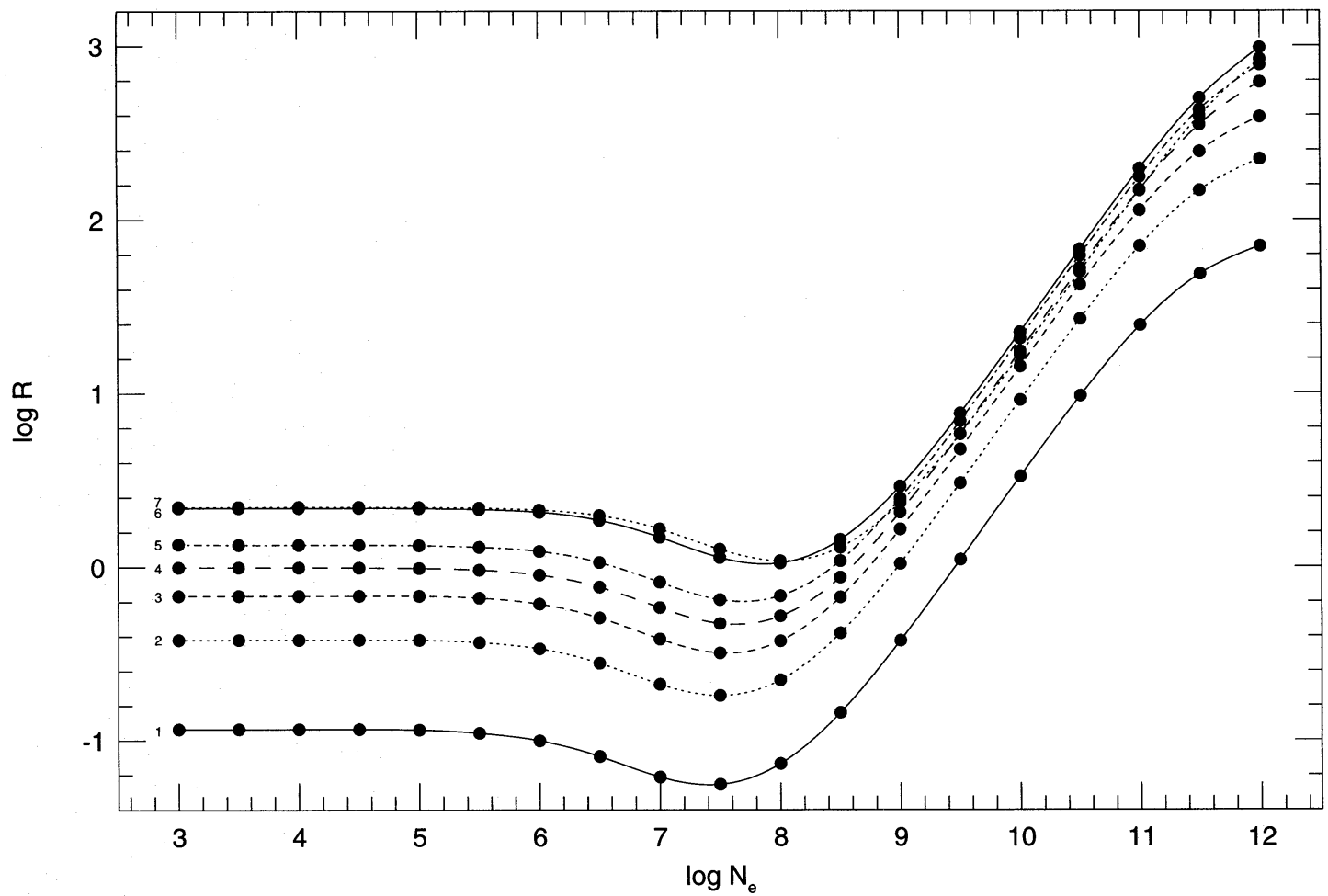

Fig. 1. - The theoretical Ne v emission line ratio $R=I\left(2 s^{2} 2 p^{2}{ }^{3} P_{1}-2 s 2 p^{3}{ }^{5} S\right) / I\left(2 s^{2} 2 p^{2}{ }^{3} P_{1}-2 s^{2} 2 p^{2}{ }^{1} S\right)=I(1137.0 \AA) / I(1574.8 \AA)$, where $I$ is in energy units, plotted as a function of logarithmic electron density $\left(N_{e}\right.$ in cm $\left.\mathrm{cm}^{-3}\right)$ at several values of electron temperature $\left(T_{e}\right)$, with curves for different $T_{e}$ labeled as follows: $1=10,000 \mathrm{~K} ; 2=15,000 \mathrm{~K} ; 3=20,000 \mathrm{~K} ; 4=30,000 \mathrm{~K} ; 5=40,000 \mathrm{~K} ; 6=100,000 \mathrm{~K} ; 7=250,000 \mathrm{~K}$. 


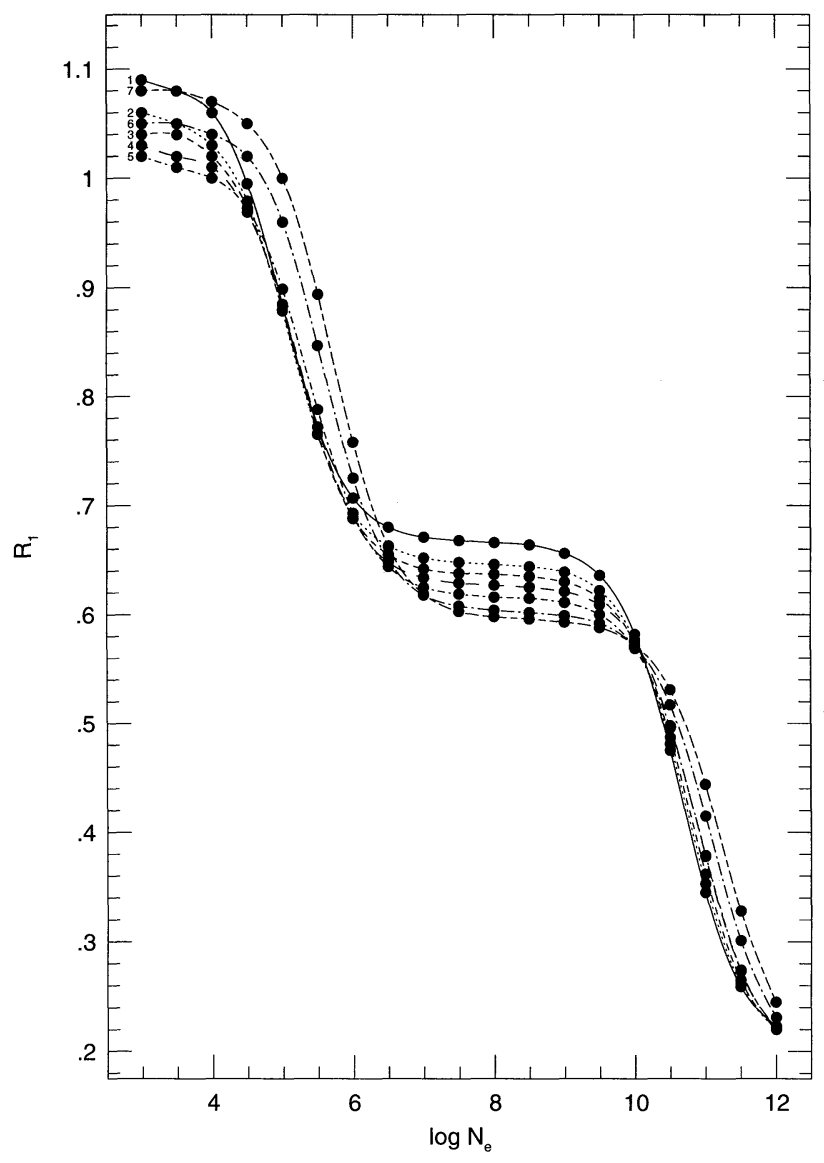

FIG. 2.-Same as Fig. 1, but for the Ne vI line ratio $R_{1}=I\left(2 s^{2} 2 p^{2} P_{3 / 2}\right.$ $\left.2 s 2 p^{24} P_{3 / 2}\right) / I\left(2 s^{2} 2 p^{2} P_{3 / 2}-2 s 2 p^{2}{ }^{4} P_{5 / 2}\right)=I(1006.1 \AA) / I(999.6 \AA)$.

levels, but with

$$
R^{\prime}=2.50 R, \quad R_{3}=1.16 R_{2} .
$$

An inspection of Figure 1 reveals that $R$ is very sensitive to variations in the electron density for $N_{e} \geq 10^{7.5} \mathrm{~cm}^{-3}$ but also varies significantly with temperature over the whole $N_{e}$ range. For example, at $N_{e}=10^{3} \mathrm{~cm}^{-3}, R$ changes by a factor of 18.8 between $T_{e}=10^{4}$ and $10^{5} \mathrm{~K}$, decreasing to a factor of 6.8 at $N_{e}=10^{10} \mathrm{~cm}^{-3}$. On the other hand, the $R_{1}$ and $R_{2}$ ratios in Ne vI vary by $\leq 12 \%$ and $\leq 17 \%$, respectively, over the same temperature interval, while also showing some $N_{e}$ sensitivity, changing by a factor of $\sim 4$ between $N_{e}=10^{3}$ and $10^{12} \mathrm{~cm}^{-3}$. Hence, in principle, the $R, R_{1}$, and $R_{2}$ ratios may be useful in determining both the electron temperature and density of the high- $T_{e}$ region of a nebula, with the Ne vI ratios primarily acting as $N_{e}$ diagnostics, and $\mathrm{Ne} \mathrm{V}$ as a $T_{e}$ diagnostic. We note that, although $R$ neither increases nor decreases monotonically with $N_{e}$, reaching a minimum in the density interval $N_{e}=10^{7}-10^{8}$ $\mathrm{cm}^{-3}$, one should be able to infer the density regime of the plasma in question using $R_{1}$ and $R_{2}$.

\section{OBSERVATIONAL DATA}

Observations of RR Tel covering the range 820-1840 $\AA$ were obtained with the HUT on 1995 March 12, during the Astro-2 space shuttle mission STS 67. Observational details have been published elsewhere (Espey et al. 1995), so they will not be included here. Relevant points of interest, however, are that the data were obtained with an exposure

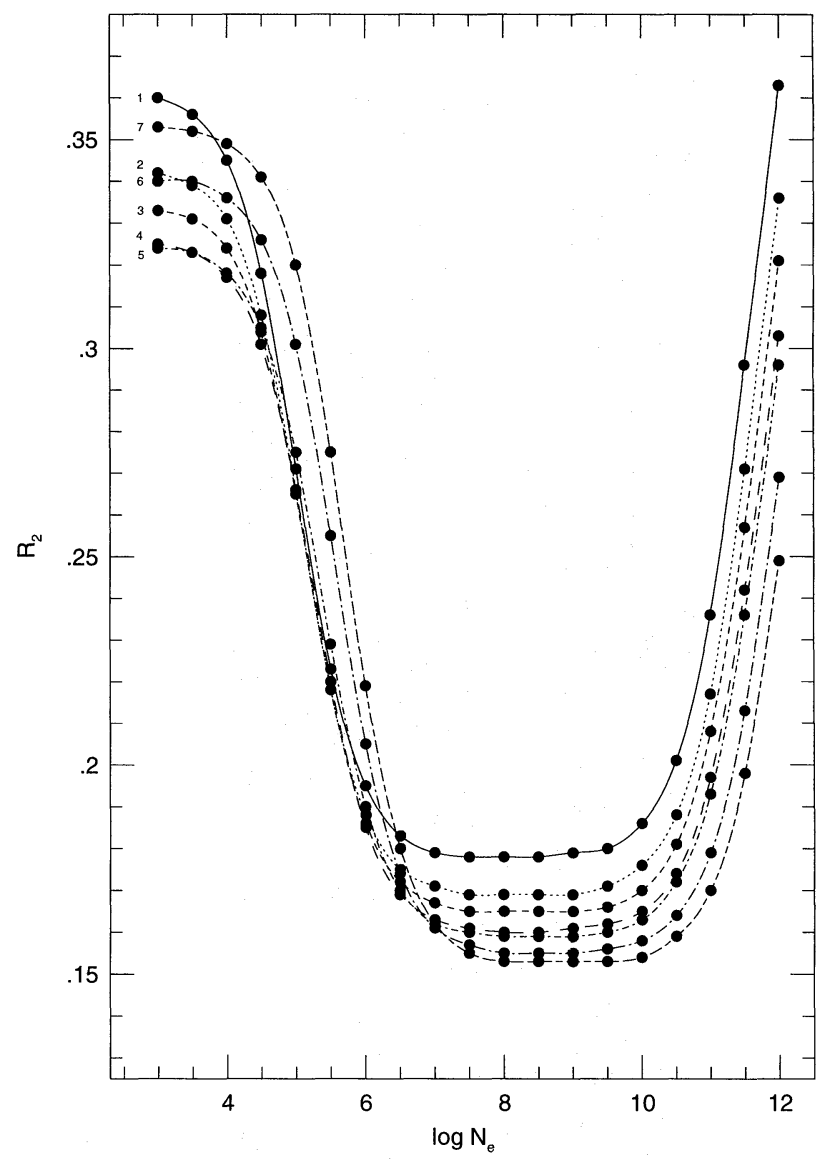

FIG. 3.-Same as Fig. 1, but for the Ne vi line ratio $R_{2}=I\left(2 s^{2} 2 p^{2} P_{3 / 2}\right.$ $\left.2 s 2 p^{2}{ }^{4} P_{1 / 2}\right) / I\left(2 s^{2} 2 p^{2} P_{3 / 2}-2 s 2 p^{2}{ }^{4} P_{5 / 2}\right)=I(1010.6 \AA) / I(999.6 \AA)$.

of $1080 \mathrm{~s}$, which resulted in signal-to-noise ratios of $\sim 6$ per pixel in the continuum around the $\mathrm{Ne} \mathrm{VI}]$ and $\mathrm{Ne} \mathrm{v}$ ] lines.

The HUT data were processed with a specially developed package of $\mathrm{IRAF}^{4}$ tasks, and the final fluxed data is believed accurate at the $\sim 5 \%$ level. The line fluxes were measured using the "SPECFIT" software developed by Kriss (1994), which uses $\chi^{2}$ minimization to obtain the bestfit Gaussian profiles to the emission lines. Table 4 provides the results of these measurements, together with their errors ( $1 \sigma$ standard deviations) as estimated by the fitting software. In the case of blends, the wavelength ratios of multiplet components were tied at their theoretical values. The line widths within each multiplet were also tied to the value of the strongest component. This was particularly important in the case of the $\mathrm{Ne} \mathrm{vI}$ lines, where the bluest components are blended with intrinsic $\mathrm{N}$ iII emission and with O I airglow because of the low altitude of the space shuttle orbit. A simple linear fit was found to give a sufficiently good representation of the local continuum under each line or blend.

Before the observed Ne line fluxes can be used for diagnostic purposes, they first need to be corrected for the effects of interstellar extinction. We chose to determine this extinction value using the 1574.8 and $2972.7 \AA\left(2 s^{2} 2 p^{2}{ }^{1} D-\right.$ $2 s^{2} 2 p^{2} S$ transition) lines of Ne v. Because this line pair

${ }^{4}$ The Image Reduction and Analysis Facility (IRAF) is distributed by the National Optical Astronomy Observatories, which is operated by the Association of Universities for Research in Astronomy, Inc. (AURA), under cooperative agreement with the National Science Foundation. 
TABLE 4

OBSERVED Ne v AND Ne Vi LiNe FluXES ${ }^{a}$

\begin{tabular}{|c|c|c|c|}
\hline Ion & Wavelength $(\AA)$ & IUE Image Number & Line Flux \\
\hline $\mathrm{Ne} v \ldots . .$. & 1137.0 & $\mathbf{H}^{\mathrm{b}}$ & $0.91 \pm 0.08^{c}$ \\
\hline $\mathrm{Ne} v \ldots . .$. & 1146.1 & $\mathbf{H}$ & $2.27 \pm 0.15$ \\
\hline $\mathrm{Ne} v \ldots \ldots$ & 1574.8 & $\mathbf{H}$ & $1.63 \pm 0.07$ \\
\hline $\mathrm{Ne} v \ldots . .$. & 1574.8 & SWP 14727 & $2.57 \pm 0.12$ \\
\hline $\mathrm{Ne} v \ldots . .$. & 2972.7 & LWR 11293 & $0.92 \pm 0.02$ \\
\hline $\mathrm{Ne}$ vi...... & 993.0 & $\mathrm{H}$ & $0.11^{\mathrm{d}}$ \\
\hline $\mathrm{Ne}$ vi....... & 997.4 & $\mathbf{H}$ & $0.42^{\mathrm{e}}$ \\
\hline $\mathrm{Ne}$ vI...... & 999.6 & $\mathrm{H}$ & $1.62 \pm 0.10$ \\
\hline $\mathrm{Ne} v i \ldots . .$. & 1006.1 & $\mathrm{H}$ & $1.01 \pm 0.07$ \\
\hline $\mathrm{Ne}$ vi...... & 1010.6 & $\mathbf{H}$ & $0.36 \pm 0.05$ \\
\hline
\end{tabular}

${ }^{a}$ Dereddened line fluxes in units of $10^{-11} \mathrm{ergs} \mathrm{cm}^{-2} \mathrm{~s}^{-1}$ derived using $E(B-V)=0.08$ (see $\S 3$ for details).

${ }^{b} \mathrm{H}$ indicates a line detected by the HUT.

c Line flux fixed at $0.40 \times I(1146.1 \AA \AA \AA)$, using the theoretical ratio.

${ }^{\mathrm{d}}$ Line flux fixed at $0.11 \times I(1006.1 \AA)$, using the theoretical ratio.

e Line flux fixed at $1.16 \times I(1010.6 \AA)$, using the theoretical ratio.

arises from a common upper level, the intrinsic line ratio is independent of density and temperature, and has a theoretical value of $I(1574.8 \AA) / I(2972.7 \AA)=2.79$ (Nussbaumer \& Rusca 1979). Because of the mild variability of the emission lines (Aufdenberg 1993), we determined the amount of extinction from data in which both lines were obtained contemporaneously. Since the $2972.7 \AA$ line lies outside the range of the HUT instrument, we use high-dispersion, largeaperture IUE spectra SWP 14727 and LWR 11293, each of 25 minutes exposure, obtained on 1981 August 1. Gaussian fits were performed using the "SPECFIT" software dis-

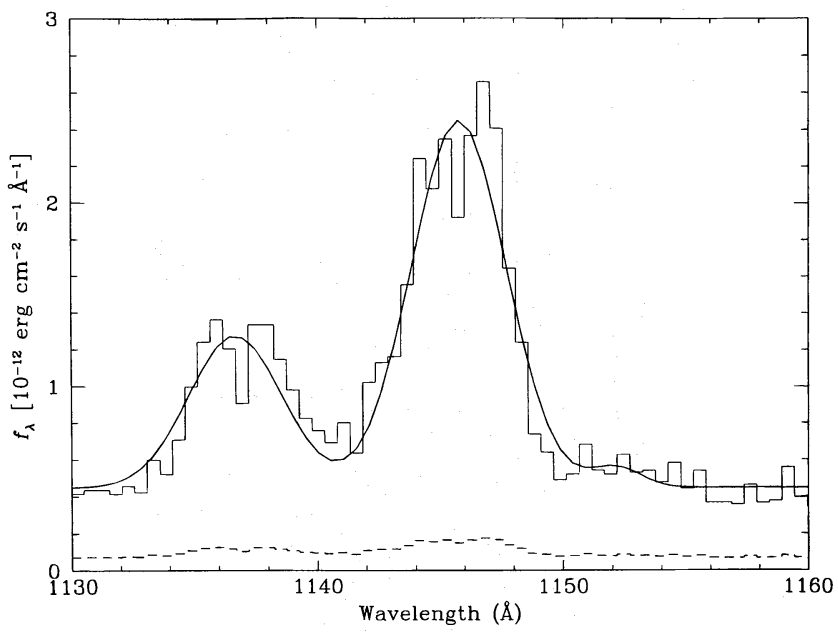

FIG. 4.-HUT spectrum of RR Tel in the 1130-1160 $\AA$ wavelength range, showing the Ne v] 1137.0 and $1146.1 \AA$ lines. Weak airglow emission lies just to the red of the $1146 \AA$ line, while the $1 \sigma$ error array derived from counting statistics is shown at the bottom of the figure. The signal-tonoise ratio in these data is $\sim 6$ in the continuum region near the $\mathrm{Ne} \mathrm{v]}$ doublet. The spectrum has not been corrected for the effects of Galactic extinction.

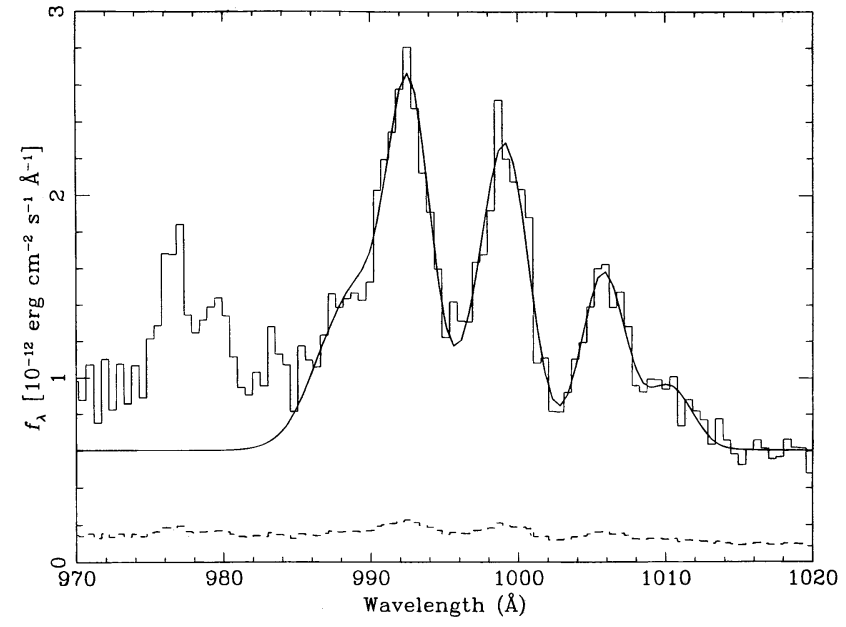

FIG. 5.-HUT spectrum of RR Tel in the 970-1020 $\AA$ wavelength range, showing the Ne VI] multiplet 993-1010 $\AA$ lines. The blended feature immediately to the blue of the Ne VI] multiplet is a combination of intrinsic $\mathrm{N}$ III emission and $\mathrm{O}_{\mathrm{I}}$ airglow, while the $1 \sigma$ error array estimated from counting statistics is plotted along the bottom of the figure. The signal-tonoise ratio in these data is $\sim 6$ in the continuum region near the $\mathrm{Ne} v \mathrm{VI}$ emission feature. The spectrum has not been corrected for the effects of Galactic extinction.

cussed above and gave an observed ratio of $2.37 \pm 0.12$. Adopting the interstellar extinction curve parameterization of Cardelli, Clayton, \& Mathis (1989) with $R_{V}=3.1$, we obtained $E(B-V)=0.08 \pm 0.03$. We note that this value of $E(B-V)$ agrees with that derived by Jordan, Mürset, \& Werner (1994) using the same method applied to lowresolution IUE spectra obtained in 1992. Our extinction estimate is also in good agreement with $E(B-V)=0.10$ found for RR Tel using other methods, such as the ratios of He II recombination lines (Penston et al. 1983) or the shape of the UV continuum (Mürset et al. 1991).

In Figures 4 and 5, we plot the HUT spectrum from 1130 to $1160 \AA$ and from 970 to $1020 \AA$, respectively, overplotted with the fitted components to illustrate the quality of the observational data. Figures 6 and 7 similarly show the $I U E$ spectra from 1570 to $1580 \AA$ and from 2960 to $2980 \AA$,

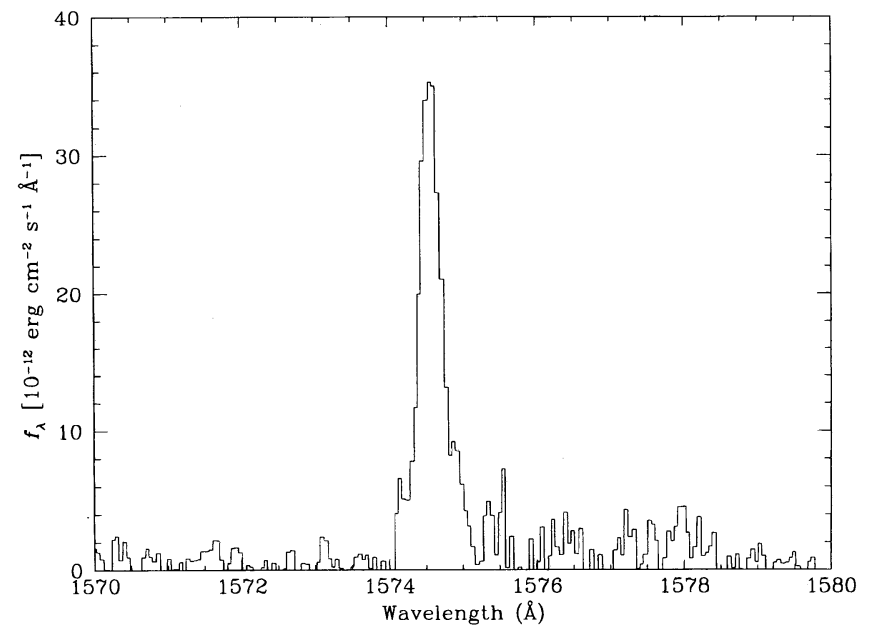

FIG. 6.-High-resolution IUE spectrum (SWP 14727) of RR Tel in the wavelength region $1570-1580 \AA$, showing the Ne v] line at $1574.8 \AA$. The data have not been corrected for Galactic extinction. 


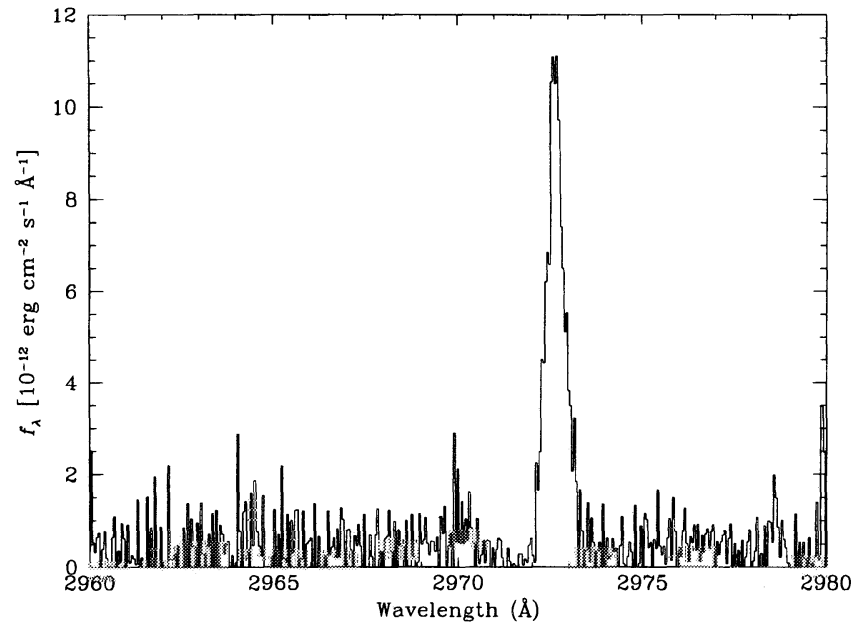

Fig. 7.-High-resolution IUE spectrum (LWR 11293) of RR Tel in the wavelength region $2960-2980 \AA$, showing the Ne v] line at $2972.7 \AA$. The data have not been corrected for Galactic extinction.

respectively, which contain the $\mathrm{Ne} v$ lines used in the extinction determination. The de-blended and extinctioncorrected $\mathrm{Ne} \mathrm{V}$ and $\mathrm{Ne}$ vi line fluxes are summarized in Table 4, with the resultant line ratios being given in Table 5 .

\section{RESULTS AND DISCUSSION}

We have calculated nebular parameters using the HUT data for the $\mathrm{Ne} \mathrm{v}$ ] and $\mathrm{Ne} \mathrm{vI}$ ] lines that should provide diagnostics on hot material in the RR Tel nebula. The presence of density and/or temperature inhomogeneities may affect the diagnostic ratios $R_{1}$ and $R_{2}$, but it should be possible to determine an "average" density and temperature for the gas responsible for the line emission. Although a complete description of the emission-line region of RR Tel is beyond the scope of this paper, we apply the temperature and density diagnostics derived here to make the first such estimates of the hot gas in this symbiotic system.

Using the $R_{1}$ and $R_{2}$ line ratios defined in $\S 2$ and summarized in Table 5, we find $\log N_{e}=8.0 \pm 2.0$ and $5.5 \pm 0.5$, respectively, where the error estimate is derived from the fit to the spectra and dominates possible errors in the atomic data (see $\S 2$ ). The larger density range derived from the $R_{1}$ ratio is due to the measured value lying in a density-insensitive range of parameter space. When these density estimates are coupled with the information provided by the Ne v] data, we find temperatures of $T_{e}<40,000 \mathrm{~K}$ and $\sim 20,000 \mathrm{~K}$ using the $R_{1}$ and $R_{2}$ ratios, respectively, thus ruling out collisional excitation for the origin of the majority of the hot gas. The Ne v] diagnostic also enables us to rule out densities above $\log N_{e} \sim 9$ predicted by the $R_{1}$ ratio, since the corresponding temperature is then $T_{e} \lesssim$ $10^{4} \mathrm{~K}$, and only weak emission is expected in this regime.

Although these results do not provide a clear-cut determination of either temperature or density for the RR Tel nebula, both diagnostic ratios should lead to similar conclusions in terms of the temperature and density of the emitting gas. We adopt a density of $\log N_{e}=6.0$, since this lies in the overlap of both error ranges, and derive a gas temperature of $T_{e}=18,000 \pm 2000 \mathrm{~K}$ from the Ne v] diagnostic curves. These values of $T_{e}$ and $N_{e}$ are in good agreement with the same parameters deduced from other high-ionization line diagnostics, which should probe the same gas. For example, Doschek \& Feibelman (1993) derived $T_{e} \simeq 20,000-25,000 \mathrm{~K}$ from the O v I(1371 $) / I(1644 \AA)$ ratio, while Hayes \& Nussbaumer (1986) found $\log N_{e} \simeq 6.2-7.0$ using $I(1602$ $\AA) / I(2423 \AA)$ in Ne IV]. This agreement provides some observational support for the theoretical $\mathrm{Ne}$ v] and $\mathrm{Ne}$ vi] diagnostics presented in this paper, and hence the atomic data used in their derivation.

\section{CONCLUSIONS}

We have used improved atomic data to calculate important diagnostics for the high-ionization ions $\mathrm{Ne} \mathrm{V}$ and $\mathrm{Ne}$ VI. Using the theoretical line ratios, together with far-UV data newly acquired by HUT, we have estimated the temperature and density of the hot nebular material in the symbiotic nova RR Tel. We find that our results are consistent with estimates made by previous workers using other line diagnostics and support models of the nebular region in which the majority of the gas is photoionized. Although RR Tel does not provide a very clear-cut case for the use of the diagnostics that we have presented here, these neon line ratios should prove useful in other astrophysical situations where hot gas is present.

F. C. M. is grateful to the European Social Fund for the award of a research studentship. The IUE archival portion of this work was supported by the IUE Data Analysis Center (IUEDAC). The HUT portion of this work was supported by NASA contract NAG8-1049. This work was financially supported by NATO travel grant CRG.930722 and the Royal Society. We thank the anonymous referee for suggestions that improved the clarity of the text and Sharon Busching for a critical reading of the manuscript.

\section{REFERENCES}

Aggarwal, K. M. 1986, ApJS, 61, 699

Aufdenberg, J. P. 1993, ApJS, 87, 337

Cardelli, J., Clayton, G., \& Mathis, J. 1989, ApJ, 345, 245

Czyzak, S. J., Keyes, C. D., \& Aller, L. H. 1986, ApJS, 61, 159

Dankworth, W., \& Trefftz, E. 1978, A\&A, 65, 93

Doschek, G. A., \& Feibelman, W. A. 1993, ApJS, 87, 331

Dufton, P. L. 1977, Comput. Phys. Commun., 13, 25

Dufton, P. L., Berrington, K. A., Burke, P. G., \& Kingston, A. E. 1978, A\&A, 62, 111

Espey, B., Schulte-Ladbeck, R. E., Kriss, G. A., Hamann F., Schmid, H. M., \& Johnson, J. J. 1995, ApJ, 454, L61

Faucher, P., Masnou-Seeuws, F., \& Prudhomme, M. 1980, A\&A, 81, 137

Flower, D. R., \& Nussbaumer, H. 1975a, A\&A, 45, 145

. 1975b, A\&A, 45, 349

Foster, V. J., Keenan, F. P., \& Reid, R. H. G. 1996a, A\&A, 308, 1009

1996b, MNRAS, submitted

Hayes, M. A., \& Nussbaumer, H. 1986, A\&A, 161, 287

Johnson, C. T., Kingston, A. E., \& Dufton, P. L. 1984, MNRAS, 207, 7P
Jordan, S., Mürset, U., \& Werner, K. 1994, A\&A, 283, 475

Keenan, F. P., Conlon, E. S., Bowden, D. A., Feibelman, W. A., \& Pradhan, A. K. 1993, ApJS, 88, 169

Keenan, F. P., Dufton, P. L., Feibelman, W. A., Bell, K. L., Hibbert, A., \& Stafford, R. P. 1994, ApJ, 423, 882

Keenan, F. P., Foster, V. J., Reid, R. H. G., Doyle, J. G., Zhang, H. L., \& Pradhan, A. K. 1995, A\&A, 300, 534

Kriss, G. A. 1994, in ASP. Conf. Ser. 61, Astronomical Data Analysis Software and Systems III, ed. D. R. Crabtree, R. J. Hanisch, \& J. Barnes (San Francisco: ASP), 437

Lennon, D. J., \& Burke, V. M. 1994, A\&AS, 103, 273

Lennon, D. J., Dufton, P. L., Hibbert, A., \& Kingston, A. E. 1985, ApJ, 294, 200

Moore, C. E. 1971, Atomic Energy Levels (NSRDS-NBS35; Washington, DC: NBS)

Mürset, U., Nussbaumer, H., Schmid, H. M., \& Vogel, M. 1991, A\&A, 248, 458

Nussbaumer, H., \& Rusca, C. 1979, A\&A, 72, 129 
Nussbaumer, H., \& Storey, P. J. 1982, A\&A, 115, 205

'I Penston, M. V., Benvenuti, P., Cassatella, A., Heck, A., \& Selvelli, P. 1983, I I I MNRAS, 202, 833

Seaton, M. J. 1964, MNRAS, 127, 191

1

1'

,

'a!

I.1
Stencel, R. E., Linsky, J. L., Brown, A., Jordan, C., Carpenter, K. G., Wing, R. F., \& Czyzak, S. 1981, MNRAS, 196, 4P

Zhang, H. L., Graziani, M., \& Pradhan, A. K. 1994, A\&A, 283, 319 